\title{
Protein Patterning with Programmable Surface Chemistry Chips
}

\author{
Y. Wang ${ }^{1}$, X. Cheng ${ }^{2}$, Y. Hanein ${ }^{1}$, A. Shastry ${ }^{1}$, D. D. Denton ${ }^{1}$, B. D. Ratner ${ }^{2,3}$, \\ K. F. Böhringer ${ }^{1}$ \\ ${ }^{1}$ Electrical Engineering, ${ }^{2}$ Bioengineering, ${ }^{3}$ Chemical Engineering \\ University of Washington, Seattle, WA
}

\begin{abstract}
Programmable surface chemistry has been achieved by depositing thermally responsive polymer (plasma polymerized $\mathrm{N}$-isopropylacrylamide, ppNIPAM) onto arrays of micro-fabricated metallic heaters. Activating a single heater causes a localized change in the device surface chemistry from non-fouling to fouling in aqueous environment. Various proteins were used to demonstrate localized immobilization of proteins on the surface of coated micro-heater arrays. Additional uses of this technique include applications such as cell patterning, tissue engineering, self-assembly, etc.
\end{abstract}

Keywords: NIPAM, protein chip, non-fouling, plasma polymerisation, heater array.

\section{Introduction}

Surface chemistry can be exploited to control a wide range of phenomena such as wetting and bio-fouling. ppNIPAM [1] is a good candidate to realize programmable surface chemistry. Among its properties is a transition from hydrophilic and non-fouling behavior at room temperature to hydrophobic and fouling behavior above its lower critical solution temperature (LCST). In addition, its LCST of $32^{\circ} \mathrm{C}$ is close to body temperature and thus suitable for protein treatment (Figure 1). These properties are due to the reversible formation and cleavage of the hydrogen bonds between $\mathrm{NH}$ or $\mathrm{C}=\mathrm{O}$ groups and surrounding water molecules with changing temperature.



Figure 1. Bovine serum albumin (BSA) immobilization tests on ppNIPAM and exposed polyethylene-terephthalate (PET) surfaces. As the temperature increases from $23^{\circ} \mathrm{C}$ to $37^{\circ} \mathrm{C}$ the BSA adsorption on ppNIPAM shows ten-fold increase. High protein adsorption on PET was observed for both temperature values. 


\section{Experiment}

In this paper we present a new method to pattern proteins using programmable surfaces consisting of ppNIPAM. Figure 2 shows the principles of our method.

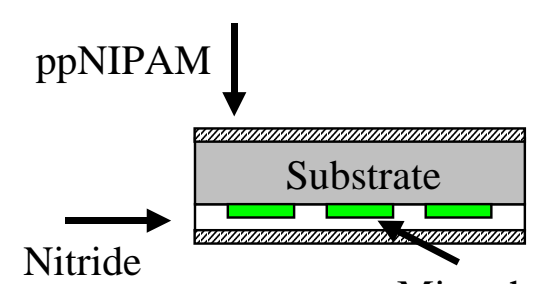

(a) Micro heater



(b)

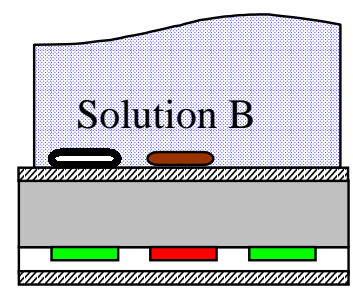

(c)

Figure 2. Schematic description of ppNIPAM devices. (a) Devices consist of microheaters on a glass slide coated with ppNIPAM. (b) Active heater (black) turns the surface fouling. Selective protein adsorption occurs exclusively on heated areas. Protein adsorption takes place on the top side of the substrate. (c) A second adsorption step with different protein solution on a different heater.

The micro-fabrication process consists of $\mathrm{CrAuCr}$ heaters (thickness $150 \mathrm{~nm}$, area $1 \times 0.8 \mathrm{~mm}$, and $40 \mu \mathrm{m}$ wide lines) deposited on a glass substrate $(180 \mu \mathrm{m})$. The heaters are passivated with sputtered silicon nitride $(400 \mathrm{~nm})$ and the entire device is then treated with ppNIPAM in a plasma deposition process [2]. This process is particularly suited for MEMS applications as it ensures very high surface coverage, excellent adhesion and good non-fouling properties at room temperature [3]. Coventor ${ }^{\mathrm{TM}}$, a fully integrated finite element simulation package, is used to simulate the electrothermal properties of the designed heaters (Figure 3). Temperature sensitive paint is used to characterize the heating profile.

When exposed to protein solution, proteins are adsorbed exclusively at heated areas. These protein patches remain immobilized even after the heater is turned off and the temperature is dropped below LCST. Repeated adsorption steps form arrays of protein patches such as those required for protein chips.

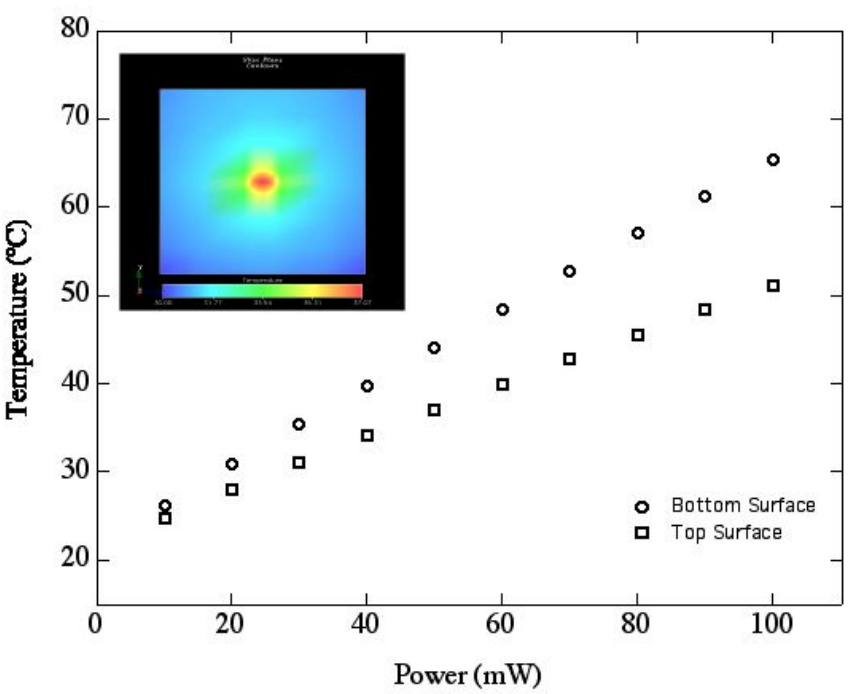

Figure 3. Simulated center temperature of top and bottom surfaces versus power (using Coventor ${ }^{\mathrm{TM}}$ ). Inset: Temperature profile of the top surface for $50 \mathrm{~mW}$. Data from actual devices agrees with values predicted for the bottom surface temperature. 


\section{Results and discussion}

A fabricated micro-heater array is shown in Figure 4a. Immunoglobulin G (IgG) test results are shown in Figure $4 \mathrm{~b}$ and $4 \mathrm{c}$. The micro heater chip was first incubated with fluorescein isothio-cyanate (FITC)-anti-BSA for 30 minutes with the upper heater turned on $(94 \mathrm{~mW})$. The upper heater was then turned off and the chip was incubated with tetramethylrhodamine isothiocyanate (TRITC)-goat-IgG with the middle heater turned on for another 30 minutes. The two fluorescence images (Figures $4 \mathrm{~b}$ and $4 \mathrm{c}$ ) were taken from the same chip with two different wavelengths. Two patches of proteins can be clearly identified on the device surface. The second protein batch did not adhere to areas already covered with anti-BSA.

The main advantage of the presented technique is the ability to perform the entire process in a wet environment, which is critical to maintain the integrity of sensitive proteins during the patterning processes. Additional major advantages

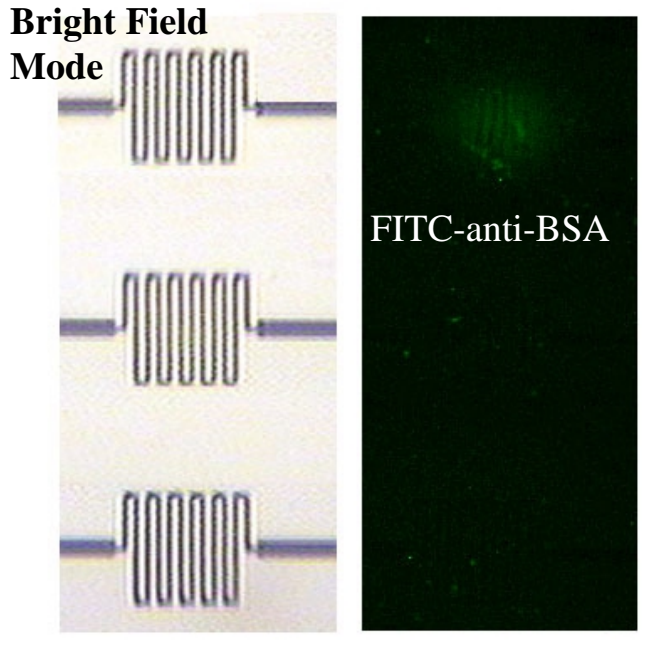

Figure 4. (a) Micro-heater array. (b) FITC-antiBSA pattern. (c) TRITC-goat-IgG pattern

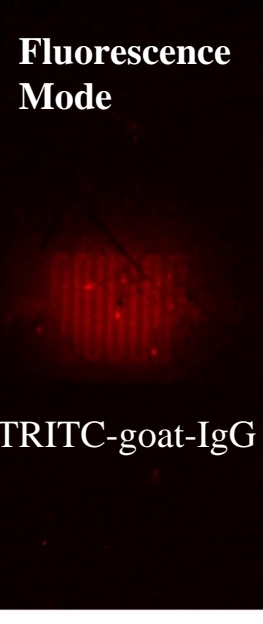

are the simple setup and the low power consumption.

\section{Conclusions}

The technique described in this paper offers a new approach to realize programmable surface chemistry devices by using micro-heater arrays to control the properties of a ppNIPAM coating. In addition to the fouling/non-fouling change, ppNIPAM also exhibits a hydrophobic/hydrophilic change in aqueous environment in response to relatively small changes in temperature, which suggests many other uses. The performance of these devices with multiple proteins, with cells and for additional MEMS and bioMEMS applications are currently investigated.

\section{References}

1. M. Heskins and J.E. Guillet, Solution properties of poly(N-isopropylacrylamide), J. Macromol. Sci. Chem., A2, pp. 1441, (1968).

2. Y.V. Pan, R.A. Wesley, R. Luginbuhl, D.D. Denton and B.D. Ratner, Plasma Polymerized N-Isopropylacrylamide: Synthesis and characterization of a smart thermally responsive coating Biomacromolecules 2, pp. 32-36, (2001).

3. Y. Hanein, Y.V. Pan, B.D. Ratner, D.D. Denton and K.F. Böhringer, Micromachined non-fouling coatings for bio-MEMS applications, Sensors and Actuators: B. Chemical, 81,pp. 49-54, (2001). 